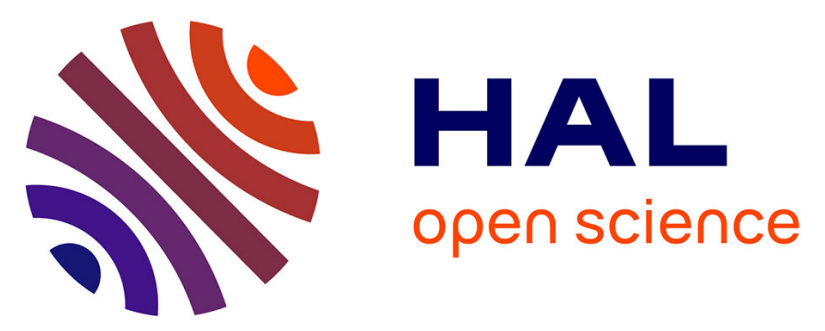

\title{
p-Doping of a Hole Transport Material via a Poly(ionic liquid) for over 20\% Efficiency and Hysteresis-Free Perovskite Solar Cells
}

Camille Geffroy, Eftychia Grana, Takeru Bessho, Samy Almosni, Zeguo Tang, Anirudh Sharma, Takumi Kinoshita, Fumiyasu Awai, Eric Cloutet, Thierry Toupance, et al.

\section{To cite this version:}

Camille Geffroy, Eftychia Grana, Takeru Bessho, Samy Almosni, Zeguo Tang, et al.. p-Doping of a Hole Transport Material via a Poly(ionic liquid) for over 20\% Efficiency and Hysteresis-Free Perovskite Solar Cells. ACS Applied Energy Materials, 2020, 10.1021/acsaem.9b01819 . hal-02456268

\section{HAL Id: hal-02456268 https://hal.science/hal-02456268}

Submitted on 27 Jan 2020

HAL is a multi-disciplinary open access archive for the deposit and dissemination of scientific research documents, whether they are published or not. The documents may come from teaching and research institutions in France or abroad, or from public or private research centers.
L'archive ouverte pluridisciplinaire HAL, est destinée au dépôt et à la diffusion de documents scientifiques de niveau recherche, publiés ou non, émanant des établissements d'enseignement et de recherche français ou étrangers, des laboratoires publics ou privés. 


\title{
p-Doping of a hole transport material via a poly(ionic liquid) for over $20 \%$ efficiency and hysteresis-free perovskite solar cells
}

\author{
Camille Geffroy, ${ }^{\mathrm{a}, \mathrm{b}, \mathrm{c}}$ Eftychia Grana, ${ }^{\mathrm{a}}$ Takeru Bessho, ${ }^{\mathrm{c}}$ Samy Almosni, ${ }^{\mathrm{d}}$ Zeguo Tang, ${ }^{\mathrm{c}}$ Anirudh \\ Sharma, ${ }^{\mathrm{a}}$ Takumi Kinoshita, ${ }^{\mathrm{d}}$ Fumiyasu Awai, ${ }^{\mathrm{d}}$ Eric Cloutet, ${ }^{\mathrm{a}}$ Thierry Toupance, ${ }^{* \mathrm{~b}}$ Hiroshi Sega- \\ wa, ${ }^{* c, d}$ and Georges Hadziioannou. ${ }^{* a}$ \\ a Laboratoire de Chimie des Polymères Organiques, LCPO UMR 5629 CNRS, Université de Bordeaux, Bordeaux INP, \\ Allée Geoffroy Saint-Hilaire, B8, F-33615 Pessac, Cedex, France. \\ b Institut des Sciences Moléculaires, ISM UMR 5255 CNRS, Université de Bordeaux, 351 Cours de la Libération, F- \\ 33405 Talence, Cedex, France. \\ c Research Center for Advanced Science and Technology, University of Tokyo, 4-6-1 Komaba, Meguro-ku, Tokyo \\ 153-8904, Japan. \\ ${ }^{\mathrm{d}}$ Graduate School of Arts and Sciences, The University of Tokyo, Komaba 3-8-1, Meguro-ku, Tokyo, 153-8902, Japan. \\ KEYWORDS. Perovskite solar cells, p-doping, poly(ionic-liquid), hole transport layer, doping mechanism.
}

\begin{abstract}
An efficient metal-free formulation of a hole transport material (HTM) based on an ionic liquid polymer is developed for n-i-p perovskite solar cells (PSCs), to address reproducibility issues related to the use of complex dopant mixtures based on lithium salts and cobalt coordination complexes. The conductivity of the HTM is thus significantly

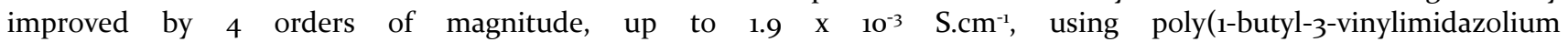
bis(trifluoromethylsulfonyl)imide) (PVBI-TFSI) as dopant. Introduced in the $\mathrm{FTO}^{\mathrm{c}-\mathrm{TiO}} / \mathrm{mp}^{-}$ $\mathrm{TiO}_{2} / \mathrm{K}_{0.05}\left(\mathrm{MA}_{0.15} \mathrm{FA}_{0.85}\right)_{0.95} \mathrm{PbI}_{2.55} \mathrm{Br}_{0.45} / \mathrm{HTM} / \mathrm{Au}$ PSC configuration, PVBI-TFSI-HTM formulation shows power conversion efficiency as high as $20.3 \%$, versus $18.4 \%$ for the standard lithium salt-HTM formulation, with considerably reduced hysteresis and excellent reproducibility. Mechanistic investigations suggest that PVBI-TFSI acts as a source of protons promoting the HTM oxidation.
\end{abstract}

\section{INTRODUCTION}

Organic-inorganic hybrid perovskite solar cells (PSCs) have gathered worldwide interest in the photovoltaic research community because of the outstanding properties of lead halide hybrid perovskite such as strong light absorption coefficients, tunable band gaps, balance charge transport and long diffusion length of the photogenerated charge carriers. ${ }^{1-3}$ Benefiting from an understanding of material properties and careful optimization of devices, remarkably high power conversion efficiencies (PCEs) over $25 \%$ have been achieved within a few years from their advent. ${ }^{4-6}$

The hole transport layer is a key component in PSCs ensuring the collection of photo-generated holes. Most efficient devices to date involve triarylamine-based HTMs, like $\quad 2,2^{\prime}, 7,7^{\prime}$-tetrakis( $N, N^{\prime}$-di-p-methoxyphenylamine)9,9'-spirobifluorene (spiro-OMeTAD) or poly(triaryl amine) (PTAA). 5, 7-9 However, these organic materials suffer from low conductivity and require p-type doping to perform similar to traditional inorganic semiconductors. In the case of spiro-OMeTAD, doping via oxidation reaction can be slowly achieved by oxygen and either light or thermal excitation. ${ }^{10}$ To accelerate this process, lithium bis(trifluoromethanesulfonyl)imide salt (LiTFSI) and tertbutylpyridine $(t \mathrm{BP})$ are usually added to the HTM precursor solution. Unlike chemical dopants, LiTFSI is a redox inactive species which does not directly react with spiroOMeTAD but participate to the oxidation reaction through lithium ions consumption." Besides, $t \mathrm{BP}$ helps solubilizing all species and improving the wetting of the HTM solution on the perovskite film being which is beneficial for the HTM morphology. The influence of oxygen on the doping level makes the doping process difficult to control, affecting the device reproducibility. Moreover, the final device stability is compromised by the ambient moisture and the use of hygroscopic salts. ${ }^{12}$

A range of other alternative materials has been investigated to enhance the conductivity of organic hole transporting materials. ${ }^{13}$ For example, the introduction of cobalt (FK102, FK209, FK269) or molybdenum complexes $\left(\mathrm{Mo}\left(\mathrm{tfd}-\mathrm{COCF}_{3}\right)_{3}, \quad \mathrm{Mo}\left(\mathrm{tfd}-\mathrm{CO}_{2} \mathrm{Me}\right)_{3}\right)$ into the spiroOMeTAD solution has been demonstrated to results in an equilibrium reaction where an electron is transferred from the spiro-OMeTAD to the complex..$^{1-17}$ In the case of copper salts $(\mathrm{CuSCN}, \mathrm{CuI})$, charge transfer results in the 
formation of a composite with spiro-OMeTAD ${ }^{18}$ Another doping strategy that has been demonstrated is the direct introduction of spiro-OMeTAD oxidized forms such as spiro $^{2+}(\mathrm{TFSI})^{2-}$ or spiro ${ }^{2+}\left(\mathrm{PF}_{6}\right)^{2-}$. In the presence of an excess of neutral spiro-OMeTAD, doubly oxidized spiroOMeTAD forms twice the amount of singly oxidized spiro-OMeTAD.19,20 Strong electron accepting compounds such as tin(IV) chloride $\left(\mathrm{SnCl}_{4}\right),{ }^{21}$ perfluorotetracyanoquinodimethane (F4TCNQ), ${ }^{22}$ tris(pentafluorophenyl)borane, ${ }^{23}$ or benzoyl peroxide, ${ }^{24}$ can also be employed as dopants for spiro-OMeTAD. These materials alone with spiro-OMeTAD were able to improve the conductivity, but for most of them, best performances were obtained together with LiTFSI and $t$ BP. The benefit of these additives is that spiro-OMeTAD oxidation can be achieved in the inert atmosphere, without involving oxygen. FK209 cobalt complex is today the best additive to the spiro-OMeTAD-LiTFSI- $t$ BP system that can achieve a higher stability and, in some processes, higher efficiencies. Few examples of alternative dopants showing superior efficiency have been reported. Mo(tfd$\left.\mathrm{COCF}_{3}\right)_{3}$ complex achieved efficiencies up to $17.8 \%$, and improved stability without employing LiTFSI nor tBP. ${ }^{17}$ Tris(pentafluorophenyl) borane doped PTAA, through the formation of an adduct, exhibited efficiency as high as 19 $\%$ with good stability. ${ }^{25}$ More recently, utilising a ZnTFSI2-FK209-tBP dopant mixture with spiro-OMeTAD, Grätzel et al. achieved highly stable and efficient PSCs, exhibiting PCE of 22.0\%. ${ }^{26}$

Another alternative to LiTFSI and $t \mathrm{BP}$ are $N$-heterocyclic ionic liquids (NHILs). Ionic liquids have the advantage to be non-volatile, to exhibit high ionic conductivity, excellent stability in air and a good ability to solvate chemicals. In the range of ionic liquids, protic and aprotic ionic liquids can be distinguished. Protic ionic liquids, with their ability to transfer protons, can be assimilated to Brønsted acid-base couples. First, Abate et al. demonstrated the ability of an imidazole-based ionic liquid (Himi-TFSI) to chemically oxidize spiro-OMeTAD in dye-sensitized solar cells. ${ }^{27}$ The mechanism proposed relies on the Brønsted acid-base property of the ionic liquid, generating a hydrogen bonding with the spiro-OMeTAD amine. Based on this study, Zhang et al. used an imidazole based ionic liquid with a pyridyl ending group which was supposed to fulfil the role of $t \mathrm{BP}$, i.e. suppress charge recombination at the $\mathrm{TiO}_{2}$ /perovskite interface in PSCs. ${ }^{28}$ In both cases, the conductivity of spiro-OMeTAD was efficiently increased without using LiTFSI nor $t$ BP. More recently, a pyridinium based ionic liquid was successfully introduced in spiro-OMeTAD achieving efficiency of $14 \%$ with improved stability. ${ }^{29}$ The doping mechanism proposed was similar to LiTFSI, where the 1-butyl-3-methylpyridinium bis(trifluoromethylsulfonyl)imide (BMPy-TFSI) participates to the oxidation reaction of spiro-OMeTAD with oxygen.

Apart from the doping ability of the materials, their integration and their effect on the PSC performance and stability should be considered. For instance, it has been reported that extrinsic ions from contact layers, such as
$\mathrm{Li}^{+}$are able to diffuse through the perovskite to the $\mathrm{TiO}_{2}$ interface. Pinholes and channels created into the layers would accelerate the degradation of the device, while the accumulation of $\mathrm{Li}^{+}$at the $\mathrm{TiO}_{2}$ /perovskite interface strongly affects charge separation and current-voltage hysteresis during PSC operation. ${ }^{30}$

In this context, we investigate an ionic liquid polymer expected to be non-diffusible to efficiently replace LiTFSI as dopant in HTM for PSCs with potassium doped organic-inorganic hybrid perovskite photo absorber. ${ }^{31,32}$ The poly(1-butyl-3-vinylimidazolium

bis(trifluoromethylsulfonyl)imide) (PVBI-TFSI) was synthesized through reversible addition fragmentation chain transfer (RAFT) controlled radical polymerization (CRP). PVBI-TFSI acts as a source of protons promoting the spiro-OMeTAD oxidation, resulting in an improved conductivity of 2 orders of magnitude compared to LiTFSI- $t$ BP doped spiro-OMeAD. Specific interactions with light and $t \mathrm{BP}$ were also evidenced. Optimized doping process with PVBI-TFSI led to PSCs exhibiting a maximum PCE of $20.3 \%$ with a negligible hysteresis and excellent reproducibility.

\section{EXPERIMENTAL SECTION}

PVBI-TFSI synthesis. 1-vinyl-3-butyl-imidazolium iodide (VBI-I) was prepared from $10.4 \mathrm{~g}$ of 1-vinyl-imidazole (99\% Alfa Aesar, o.11 mol, 1 equ.) mixed to $255 \mathrm{~mL}$ of ethyl acetate (99.5\% Sigma Aldrich, $20 \% \mathrm{w} / \mathrm{v}$ ) in a $500 \mathrm{~mL} \mathrm{3}^{-}$ necked flask cooled with an ice bath under nitrogen. Then, $40.7 \mathrm{~g}$ of 1-iodo-butane (99\% Alfa Aesar, 0.22 mol, 2 equ.) were added slowly through a septum. The solution was refluxed at $85^{\circ} \mathrm{C}$ for $20 h$. The obtained VBI-I was washed by triturations with diethylether and dried at $50^{\circ} \mathrm{C}$ under vacuum overnight. Poly(1-butyl-3vinylimidazolium iodide) (PVBI-I) was then prepared through RAFT polymerization of VBI-I. $2 \mathrm{~g}$ of VBI-I (7.2 mmol, 1 equ.), $93 \mathrm{mg}$ of O-ethyl-S-(1-ethoxycarbonyl) ethyldithiocarbonate (CTA, Sigma Aldrich, $0.42 \mathrm{mmol}$, 0.06 equ.) and $34 \mathrm{mg}$ of 2,2'-azobis(2-methylpropionitrile) (AIBN, 98\% Sigma Aldrich, 0.21 mmol, o.03 equ.) were mixed in $10.5 \mathrm{~mL}$ of anhydrous $\mathrm{N}, \mathrm{N}$-dimethyl-formamide $(\mathrm{DMF}, 20 \% \mathrm{w} / \mathrm{v})$ in a $50 \mathrm{~mL}$ round bottom flask equipped with a magnetic stirrer. After 3 freeze-vacuum-thaw cycles, the mixture was stirred under nitrogen atmosphere at $60^{\circ} \mathrm{C}$ and the polymerization was stopped after $24 \mathrm{~h}$ by dipping the polymerization flask into liquid nitrogen. Anion exchange was finally performed on PVBI-I to obtain PVBI-TFSI. $4 \mathrm{~g}$ of PVBI-I (14 mmol, 1 equ.) were solubilized in methanol $(20 \% \mathrm{w} / \mathrm{v})$. A solution of LiTFSI in THF ( $31 \mathrm{mmol}, 2.2$ equ., $75 \% \mathrm{w} / \mathrm{v}$ ) was then slowly added to the mixture under nitrogen. The solution was stirred for $24 \mathrm{~h}$. After evaporating methanol and THF, the obtained solid was immersed in water at $40^{\circ} \mathrm{C}$ for $\mathrm{th}$ and rinsed three times to remove the excess of LiTFSI and iodide. The final polymer had a molecular mass of 7350 g.mol-1. ${ }^{1} \mathrm{H}$ NMR spectrum and GPC/SEC of the polymer are given in Figure $\mathrm{S}_{1}$ and $\mathrm{S}_{2}$, respectively. 
Fabrication of solar cells. All materials were employed as received. Perovskite materials, including FAI (> 99 \%) and $\mathrm{MABr}$ (> $98 \%$ ) were purchased from Dyesol, and $\mathrm{PbI}_{2}$ (99.99\%) and $\mathrm{PbBr}_{2}$ (> $98 \%$ ) from Kojundo Chemical Laboratory Co. KI (> 99.5 \%) and magnesium(II) bis(trifluoromethanesulfonyl)imide $\left(\mathrm{Mg}(\mathrm{TFSI})_{2}\right)$ were obtained from TCI. Titanium diisopropoxide bis(acetylacetonate) (75 wt. \% in isopropanol) (TiAcAc), lithium bis(trifluoro methanesulfonyl)imide (LiTFSI) and tert-butylpyridine $(t \mathrm{BP})$ were bought from Sigma-Aldrich. The $24 \mathrm{~nm}$ nano- $\mathrm{TiO}_{2}$ paste (PST-24NR) was purchased from JPG C\&C and the spiro-OMeTAD from Merck. All solvents were obtained from Wako Pure Chemical Industries. FTO glass substrates (sheet resistance of $\approx 10 \Omega / \square$, Nippon Sheet Glass Co.) were first laser etched and successively cleaned 10 minutes in an ultrasonic bath in water, detergent, acetone and ethanol, and by excimer UV cleaner. A solution made of TiAcAc mixed with 3 mol.\% of LiTFSI and $3 \mathrm{~mol} \%$ of $\mathrm{Mg}(\mathrm{TFSI})_{2}$ in ethanol was deposited by spray pyrolysis at $430^{\circ} \mathrm{C}$ to form a $40-\mathrm{nm}$ compact- $\mathrm{TiO}_{2}$ layer. The next layers were deposited in a dry room with a dew point temperature at $-30^{\circ} \mathrm{C}$. A 150 $\mathrm{nm} \mathrm{mp}-\mathrm{TiO}_{2}$ layer was made by spin coating a solution of 24-nm- $\mathrm{TiO}_{2}$ paste diluted in ethanol (14 wt.\%), followed by 2-step annealing, at $125^{\circ} \mathrm{C}$ for $5 \mathrm{~min}$ and $550^{\circ} \mathrm{C}$ for 15 min. The layer was then doped with a LiTFSI solution in acetonitrile (o.1 $\mathrm{M})$ deposited by spin coating and annealed at $100^{\circ} \mathrm{C}$ for $5 \mathrm{~min}$ and $430^{\circ} \mathrm{C}$ for $30 \mathrm{~min}$. The perovskite precursor solution was made by dissolving $\mathrm{MABr}$, $\mathrm{PbBr} 2, \mathrm{PbI}_{2}$, and FAI (1:1:5.75:5.45 mol) in a mixture of DMSO and DMF (1:4 vol). 5 mol.\% of KI, which was previously dissolved in DMSO (1.5 M), was added to the perovskite solution. The solution was spin-coated at a speed of $4000 \mathrm{rpm}$ with a slope of $1 \mathrm{~s}$. $0.5 \mathrm{~mL}$ of chlorobenzene were dropped after $30 \mathrm{~s}$ of spin while the substrate was still spinning. This anti-solvent method led to a dark, smooth and homogeneous perovskite layer which was annealed at for $160^{\circ} \mathrm{C}$ for 15 minutes. Standard LiTFSIdoped spiro-OMeTAD solution is made by dissolving spiro-OMeTAD in chlorobenzene (o.06 M), adding 0.03 $\mathrm{M}$ of LiTFSI solution in acetonitrile $(1.85 \mathrm{M})$ and $0.20 \mathrm{M}$ of $t$ BP. For the PVBI-TFSI-doped spiro-OMeTAD, LiTFSI was replaced by PVBI-TFSI without being previously dissolved. HTM solutions were spin coated at $4000 \mathrm{rpm}$ after substrates have cooled down. Layers were annealed at $70^{\circ} \mathrm{C}$ for $10 \mathrm{~min}$ in the case of LiTFSI-doped spiroOMeTAD. Substrates were left overnight in a dry room. Finally, the 8o-nm top gold electrode was evaporated.

Sample preparation for UPS analysis. To avoid charging of samples, thin films of spiro-OMeTAD were deposited on silicon by diluting the concentration of precursor solution to $25 \%$ of that used for device fabrication. Doping was realized by incorporating PVBI-TFSI and $t \mathrm{BP}$ in 1:1:6.6 proportion.

Sample preparation for conductivity measurements. In-plane devices were prepared to measure the conductivity of HTMs. 300-nm thick HTM layers were spin coated on top a poly(1-vinyl-1,2,4-triazole) films (20 nm) on glass substrates. Gold electrodes were evaporated on top of the
HTM. Conductivities were calculated as $\sigma=\mathrm{L} /(\mathrm{Rwt})$, where $\mathrm{L}$ is the channel length $(0.05 \mathrm{~mm})$, w the channel width $(2.5 \mathrm{~mm})$, $\mathrm{t}$ the film thickness $(300 \mathrm{~nm})$ and $\mathrm{R}$ the film resistance.

Characterizations. UV/vis absorption spectra were recorded on a Shimadzu UV-36oo spectrometer. Ionization potentials were measured with a Riken Keiki AC-3 photoelectron spectrometer in air. UPS measurements were performed in an ultra-high vacuum chamber built by SPECS (Germany), using a low intensity UV light (He I line) with excitation energy of $21.21 \mathrm{eV}$. The samples were prepared in a nitrogen glovebox and transferred to the analysis chamber via a mechanized linear transfer line $\left(10^{-}\right.$ 1o mbar range) connected to the nitrogen glovebox, with no exposure to an ambient air atmosphere. The spectra of photo-emitted electrons were recorded at pass energy of 2 $\mathrm{eV}$ with a hemispherical analyser (Phoibos 100). I-V curves were measured under AM 1.5G (100 mW.cm-2) using $450 \mathrm{~W}$ xenon as the light source (YSS-8oA, Yamashita Denso). The light intensity was calibrated using a standard Si photodiode of BS-520 (Bunkoukeiki Co). The scan was carried out between -0.2 V and 1.2 V at $100 \mathrm{mV} . \mathrm{s}$ 1. The active area was defined by a black mask of 0.181 $\mathrm{cm}^{2}$. EQE spectra were recorded on an EQE system (CEP2oooMLQ, Bunkoukeiki Co.).

\section{RESULTS AND DISCUSSION}

In the following discussion, Li-spiro and PVBI-spiro refer respectively to LiTFSI and $t \mathrm{BP}$ doped spiro-OMeTAD and to PVBI-TFSI and $t$ BP doped spiro-OMeTAD. Compositions are expressed as molar ratios $x: y: z$, where $x$ corresponds to spiro-OMeTAD, y to LiTFSI or to PVBI-TFSI (moles of monomer), and $\mathrm{z}$ to $t \mathrm{BP}$. Herein, the 1:0.5:3.3 Lispiro composition leading to the best photovoltaic (PV) performances was used as standard composition.

Thin film properties. The thin film conductivities of spiro-OMeTAD depending on the dopant nature, the dopant concentration, and the light irradiance were investigated by performing two-probe conductivity measurements following a previously reported method. ${ }^{11}$ Current-voltage $(\mathrm{I}-\mathrm{V})$ curves were recorded without and with exposure (1h3o under) to AM1.5 illumination (100 mW.cm $\left.{ }^{2}\right)$, respectively. The conductivity of spiro-OMeTAD was improved from $9.4 \times 10^{-8}$ to $6.0 \times 10^{-6}{\mathrm{~S} . \mathrm{cm}^{-1}}$ with LiTFSI as dopant in ambient air atmosphere and enhanced to $1.1 \mathrm{x}$ $10^{-5}{\mathrm{~S} . \mathrm{cm}^{-1}}$ after 1 h3o of irradiation (Figure $\mathrm{S}_{3}$, Table 1). These results were in accordance with the literature data. ${ }^{11}$ Conductivity was greatly improved to $1.6 \times 10^{-5} \mathrm{~S}^{-\mathrm{cm}^{-1}}$ when LiTFSI was replaced by PVBI-TFSI. After light exposure (AM1.5), the best conductivity of $1.9 \times 10^{-3}{\mathrm{~S} . \mathrm{cm}^{-1}}^{-1}$ was recorded for the 1:1:6.6 formulation. The correlation between the increase in conductivity and the oxidation of spiro-OMeTAD was studied by recording the absorption spectra of thin films processed using the same conditions. The feature at $390 \mathrm{~nm}$ (Figure $\mathrm{S}_{4}$ ) corresponds to pristine spiro-OMeTAD. Two other distinct peaks were found at around $450 \mathrm{~nm}$ and $600 \mathrm{~nm}$. Based on calculated absorption spectra of different spiro-OMeTAD forms published 
by Abate et al., peaks at $450 \mathrm{~nm}$ and $580 \mathrm{~nm}$ can be assigned to spiro-OMeTAD-NH., while peaks at $500 \mathrm{~nm}$ and $690 \mathrm{~nm}$ are attributed to spiro-OMeTAD $\cdot{ }^{+} .{ }^{27}$ With a simultaneous increase of PVBI-TFSI and $t \mathrm{BP}$ or a decrease of $t \mathrm{BP}$ concentration, pristine-spiro-OMeTAD peak at 390 $\mathrm{nm}$ decreased while peaks associated with spiroOMeTAD-NH. was red shifted, up to the spiro-OMeTAD.+ form, in the case of 1:1:3.3 concentration. These results confirm that PVBI-TFSI promotes the oxidation of spiroOMeTAD even more readily when lower amounts of $t \mathrm{BP}$ is present. ${ }^{33}$ Finally, using Li-spiro in the same concentration, the peaks associated with oxidized spiro-OMeTAD were blue-shifted, suggesting a weaker oxidation ability in the presence of LiTFSI compared to PVBI-TFSI (Figure $\mathrm{S}_{4 \mathrm{C}) \text {. }}$

Table 1 Average conductivities (4 films) and standard deviation of Li-spiro and PVBI-spiro thin films.

\begin{tabular}{|c|c|c|c|c|}
\hline Dopant & $\begin{array}{c}\text { Molar } \\
\text { equiva- } \\
\text { lence }\end{array}$ & $\begin{array}{c}\text { Conductivity } \\
\text { before illumi- } \\
\text { nation } \\
{[\text { S.cm-1] }}\end{array}$ & $\begin{array}{c}\text { Conductivity } \\
\text { after illumi- } \\
\text { nation } \\
{[\text { S.cm-1] }}\end{array}$ & $\begin{array}{c}\text { HOMO } \\
\text { level from } \\
\text { PESA } \\
{[\mathrm{eV}]}\end{array}$ \\
\hline None & $1: 0: 0$ & $9.4 \pm 2.4$ E-08 & $2.5 \pm 0.2$ E-06 & -5.25 \\
\hline LiTFSI & $1: 0.5: 3 \cdot 3$ & $6.0 \pm 0.1$ E-06 & $1.1 \pm 0.2$ E-05 & -5.35 \\
\hline $\begin{array}{c}\text { PVBI- } \\
\text { TFSI }\end{array}$ & $1: 0.5: 3 \cdot 3$ & $1.6 \pm 0.8$ E-05 & $3.8 \pm 1.0$ E-04 & -5.30 \\
\cline { 2 - 6 } & $1: 1: 3 \cdot 3$ & $6.1 \pm 2.2$ E-05 & $9.1 \pm 5.0$ E-04 & b) \\
\cline { 2 - 6 } & $1: 1: 6.6$ & $7.8 \pm 0.3$ E-05 & $1.9 \pm 0.5$ E-03 & -5.35 \\
\cline { 2 - 6 } & $1: 1: 13.2$ & $2.6 \pm 2.2$ E-05 & $1.1 \pm 0.3$ E-03 & b) \\
\cline { 2 - 6 } & $1: 1.5: 9.9$ & $1.1 \pm 0.6$ E-04 & $4.4 \pm 2.4$ E-03 & -5.41 \\
\hline
\end{tabular}

a) $\mathrm{x}, \mathrm{y}$ and $\mathrm{z}$ correspond to spiro-OMeTAD, LiTFSI or PVBI-TFSI (moles of monomer), and $t \mathrm{BP}$ respectively; $\mathrm{b}$ ) Not measured.

Importantly, the conductivity was found to increase with irradiance and reached a maximum when the ratio of Spiro, PVBI-TFSI and $t \mathrm{BP}$ concentrations was 1:1:6.6. This effect can be explained by the topography of the films

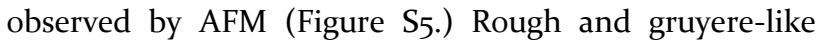
films were formed when adding more PVBI-TFSI, whereas morphology was improved when adding more $t \mathrm{BP}$. So the film conductivity is either limited by the morphology or by the oxidation level of the spiro-OMeTAD, which are both controlled by the concentration of dopants.

Since doping allows to control Fermi level position of semiconductors and facilitates hole injection, HOMO levels of pristine spiro-OMeTAD, Li-spiro and PVBI-spiro were measured by photoelectron spectroscopy in air (PESA) (Table 1) and by UPS in inert atmosphere. In air, the pristine spiro-OMeTAD HOMO level was $-5.25 \mathrm{eV}$. Addition of dopants led to down-shifted HOMO levels of $-5.35 \mathrm{eV}$ with LiTFSI and from -5.30 to $-5.41 \mathrm{eV}$ with PVBITFSI when increasing the concentration. By UPS, the work function of pristine spiro-OMeTAD and PVBI-spiro were determined as the difference between the excitation energy and the length of the spectrum. 34 Pristine spiroOMeTAD deposited on a silicon wafer exhibited a work function of $4.15 \mathrm{eV}$ and a VBM of $0.80 \mathrm{eV}$. Upon the addition of PVBI-TFSI, spiro-OMeTAD work function increased to $4.50 \mathrm{eV}$ and the VBM shifted to $0.60 \mathrm{eV}$ (Figure
1). Through the deeper HOMO level closer to the Fermi level of PVBI-spiro, this study indicates that PVBI-TFSI promotes p-doping of spiro-OMeTAD. Doped spiroOMeTAD is expected to have a better energy level alignment with the underlying perovskite layer and hence better hole injection.

Photovoltaic performances. PVBI-TFSI doped spiroOMeTAD was introduced in mesoscopic PSCs using the following n-i-p device configuration: $\mathrm{FTO} / \mathrm{c}-\mathrm{TiO}_{2} / \mathrm{mp}-$ $\mathrm{TiO}_{2} / \mathrm{K}_{\text {o.05 }}\left(\mathrm{MA}_{0.15} \mathrm{FA}_{0.85}\right)_{0.95} \mathrm{PbI}_{2.55} \mathrm{Br}_{\text {o. } 45} / \mathrm{HTM} / \mathrm{Au}$. Effects of PVBI-TFSI and $t \mathrm{BP}$ concentrations on the PV performance were investigated and compared to the standard Li-spiro. SEM cross sections revealed homogeneous perovskite layer in both devices (Figure S6). The highest power conversion efficiency (PCE) of $20.33 \%$ was recorded for devices incorporating PVBI-spiro as compared to that of $18.44 \%$ obtained for the standard devices utilizing Li-spiro (Figure 2a). This increase in efficiency is due to a simultaneous increase in all device parameters such as, $\mathrm{V}_{\text {oc }}$ from $1.10 \mathrm{~V}$ to $1.16 \mathrm{~V}$, fill factor (FF) from 0.73 to 0.76 and short-circuit current $\left(\mathrm{J}_{\mathrm{sc}}\right)$ from $22.45 \mathrm{~mA} . \mathrm{cm}^{-2}$ to 22.99 $\mathrm{mA} \cdot \mathrm{cm}^{-2}$, when LiTFSI was replaced by PVBI-TFSI as the dopant for spiro-OMeTAD. The increase in $\mathrm{J}_{\mathrm{sc}}$ is also confirmed by the IPCE measurements, where a current of $22.36 \mathrm{~mA} . \mathrm{cm}^{-2}$ was extracted for PVBI-spiro whereas 20.94 $\mathrm{mA} . \mathrm{cm}^{-2}$ was extracted for Li-spiro PSCs (Figure $2 \mathrm{~b}$ ). In previous report, we observed that the device with hysteresis in IV curve shows slow photo-current response. ${ }^{32}$ This indicated that the current of solar cell with $\mathrm{Li}^{+}$doped HTM in IPCE measurement is not saturated enough due to the I-V hysteresis (Figure 2a) that lead to the lower IPCE and integrated current value than measuring under 1 sun irradiation. Reproducibility on 20 cells was as good for Li-spiro and PVBI-spiro PSCs and confirmed the higher performances of PVBI-TFSI (Figure 2c). Higher PV performances and especially $\mathrm{J}_{\mathrm{sc}}$ and the FF of PVBI-TFSI devices can be attributed to the improved conductivity of HTM film and the lower sheet resistance in the device.

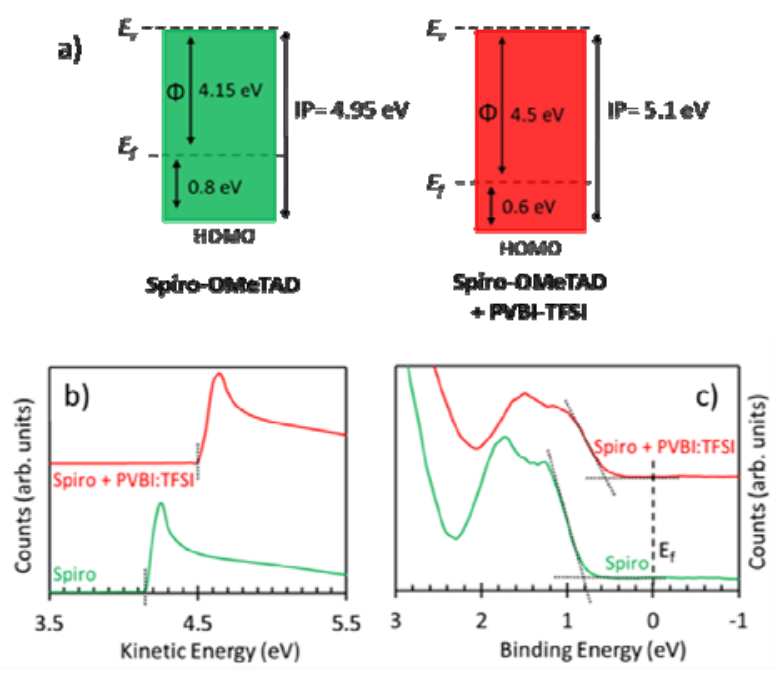

Figure 1. a) Band structure diagram based on UPS of spiro-OMeTAD and PVBI-spiro. b) Secondary electron cut-off and c) valence band region of UPS spectra. 
a)
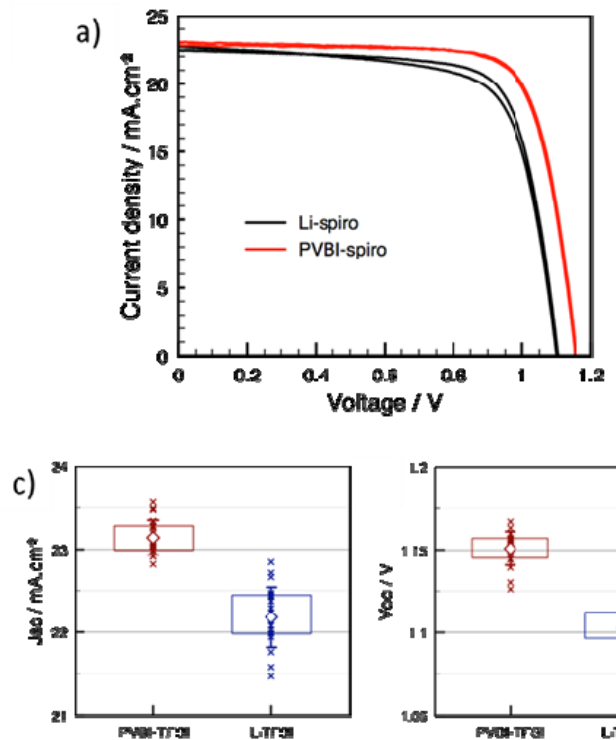
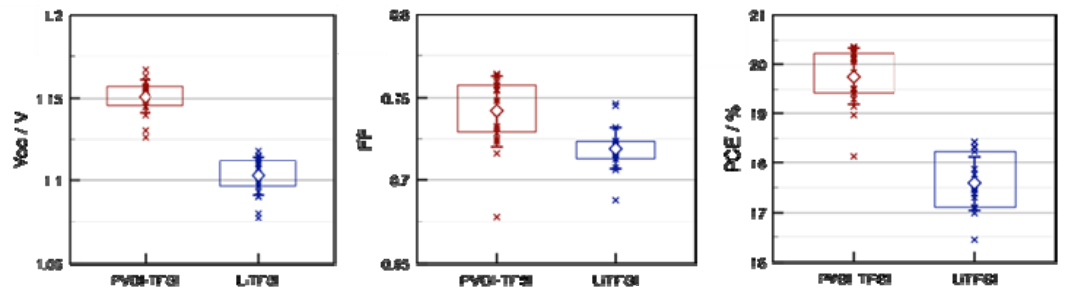

Figure 2. a) Best I-V curves recorded for Li-spiro and PVBI-spiro PSCs and b) their corresponding IPCE. c) Statistical representations of $\mathrm{J}_{\mathrm{sc}}, \mathrm{V}_{\mathrm{oc}}$, FF and PCE of PSCs containing PVBI-TFSI or LiTFSI.

A major problem remaining in PSCs to correctly assess the PCE is the hysteresis in I-V curves. I-V curves are affected by the scan direction, the scan rate and the preconditioning of the cells. This had been explained by the relatively slow ion migration of the perovskite crystal under operating conditions and by the presence of trap states in the perovskite crystal and at the ETL/perovskite and perovskite/HTM interfaces. ${ }^{35}$ Usually in n-i-p PSCs, hysteresis is characterized by lower $\mathrm{V}_{\mathrm{oc}}$ and $\mathrm{FF}$ for the forward bias and can be quantified by the hysteresis index defined as $\mathrm{HI}=\left(\mathrm{PCE}_{\text {reverse }}-\mathrm{PCE} \mathrm{E}_{\text {forward }}\right) / \mathrm{PCE}_{\text {reverse. }}$ HIs were found to be -0.007 and 0.002 in PVBI-spiro and in $\mathrm{Li}$ spiro, respectively (Table S1). Additional to enhance PCE, PVBI-spiro based PSCs showed negligible hysteresis.

Photoluminescence (PL) and time resolved photoluminescence (TRPL) measurements were performed to investigate charge transfer properties and relaxation processes at the interfaces. When the device is irradiated from the FTO side, PL reflects processes occurring close to the perovskite $/ \mathrm{TiO}_{2}$ interface, whereas when the device is irradiated from the HTM side, it reflects processes occurring close to the perovskite/HTM interface. For PVBIspiro, PL occurred at $782 \mathrm{~nm}$ on both $\mathrm{TiO}_{2}$ and HTM side, while it was remarkably red-shifted from 782 for the $\mathrm{TiO}_{2}$ side to $794 \mathrm{~nm}$ for the HTM side in the case of Li-spiro (Figure $\mathrm{S}_{7}$ ). One reason could be the effect of $\mathrm{Li}^{+}$diffusion from the HTM to the $\mathrm{TiO}_{2}$ surface which have been pointed out before to create defects in the perovskite layer, resulting in a red-shift of the perovskite bandgap. ${ }^{29}$ The diffusion of $\mathrm{Li}+$ from the HTM side is non-Fickian diffusion, leading to high $\mathrm{Li}+$ concentration on the $\mathrm{TiO}_{2}$ surface in the device due to the high affinity between $\mathrm{TiO}_{2}$ and $\mathrm{Li}^{+}$ions. ${ }^{30}$ The 150 -nm-thick perovskite/mesoporous- $\mathrm{TiO}_{2}$ layer has a specific surface area that is more than two orders of magnitude larger than that of the HTM/perovskite interface; therefore, the defect sites of perovskite are more distributed on the FTO side. Considering emission spectra on HTM side, PL peak appeared at the same position for each dopant suggesting that the local perovskite bandgap is not affected. It should be noted that emission decay curves irradiated from $\mathrm{TiO}_{2}$ side are very similar in Li-spiro and PVBI-spiro solar cells (Figure 3). From HTM side, emission decay of Li-spiro quenched faster than PVBI-spiro, suggesting that hole injection is improved with Li-spiro. However, according
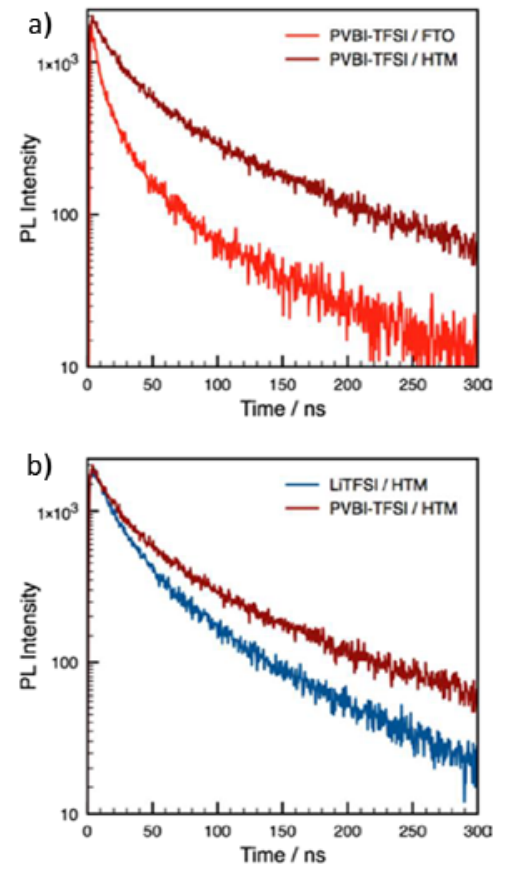

Figure 3. TRPL of PSCs containing a) PVBI-spiro and b) Li-spiro.

to deconvolution analysis, at least three decay mechanisms were involved (Table S2). The faster parameter $\tau 1$ is considered to reflect hole injection into the HTM since it is shorter than the emission lifetime of perovskite on 
glass. Thus, parameters $\tau_{1}$ of 14.8 and 17.4 ns were found for PVBI-spiro and Li-sipro, respectively.

As a consequence, hole injection should be more efficient in PVBI-spiro. Finally, the longer charge carrier lifetime in PVBI-spiro could be due to less non-radiative/more radiative recombinations into the perovskite as compared to Li-spiro, which also explains the reduced hysteresis in PVBI-spiro PSCs. To confirm this interpretation, photoluminescence quantum efficiency (PLQE) could be performed. If higher PLQE is found in the PVBI-spiro PSC, it would mean that there is less non-radiative recombinations in the PVBI-spiro devices. ${ }^{36}$
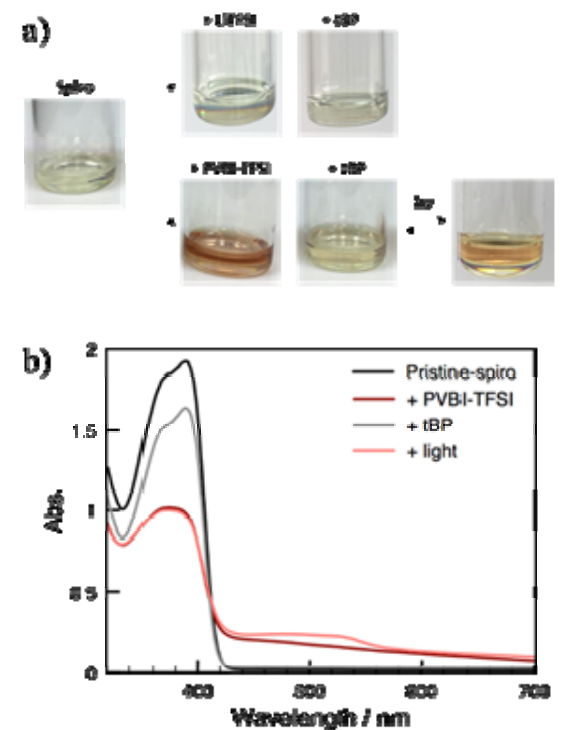

Figure 4. a) Coloration of spiro-OMeTAD solution vs. addition of LiTFSI, PVBI-TFSI and $t B P$, and $b)$ their corresponding absorption spectra.

Doping mechanism. Finally, the doping mechanism of spiro-OMeTAD by PVBI-TFSI including the effect of $t \mathrm{BP}$ and irradiance was studied. Spiro-OMeTAD oxidation can be simply observed by a color change in the precursor solution (Figure 4a). Without any dopant or upon the addition of LiTFSI and $t \mathrm{BP}$, spiro-OMeTAD solution is almost transparent. When PVBI-TFSI was incorporated to the spiro-OMeTAD, the solution turned immediately red. This color change was suppressed when $t \mathrm{BP}$ was further added. After exposition to ambient light for some minutes, the solution turned red again, and this last process was temporary reversible. Ageing or over light exposure kept the solution reddish. To understand the mechanisms governing the spiro-OMeTAD oxidation process and their dependency to $t \mathrm{BP}$ and irradiance, absorption spectra were recorded in solution for each step (Figure 4b). Pristine spiro-OMeTAD showed an absorption peak at $390 \mathrm{~nm}$ which remarkably decreased upon the addition of PVBI-TFSI. This phenomenon was associated with the concomitant appearance of the oxidized spiro-OMeTAD species feature at around $510 \mathrm{~nm}$. After the addition of $t \mathrm{BP}$ only the pristine spiro-OMeTAD peak at $390 \mathrm{~nm}$ remained with a reduced intensity, suggesting that part of spiro-OMeTAD has reacted to form a species not detecta- ble in the visible range. After irradiation for 30 min under AM1.5 $100 \mathrm{~mW} . \mathrm{cm}^{-2}$, the oxidized spiro-OMeTAD species were formed while the intensity of the pristine spiroOMeTAD peak was further reduced. These results agree with absorption spectra recorded in thin films and proved that spiro-OMeTAD oxidation occurs in the presence of PVBI-TFSI.

The oxidation of spiro-OMeTAD by NHILs has already been described in the literature..$^{28,29}$ The proposed mechanism considered the NHIL as a weak Brønsted acid, able to protonate spiro-OMeTAD and form a carbene. However, $N$-heterocyclic carbenes are usually formed by deprotonation of azolium salts with a strong base in dry and air-free conditions. ${ }^{37}$

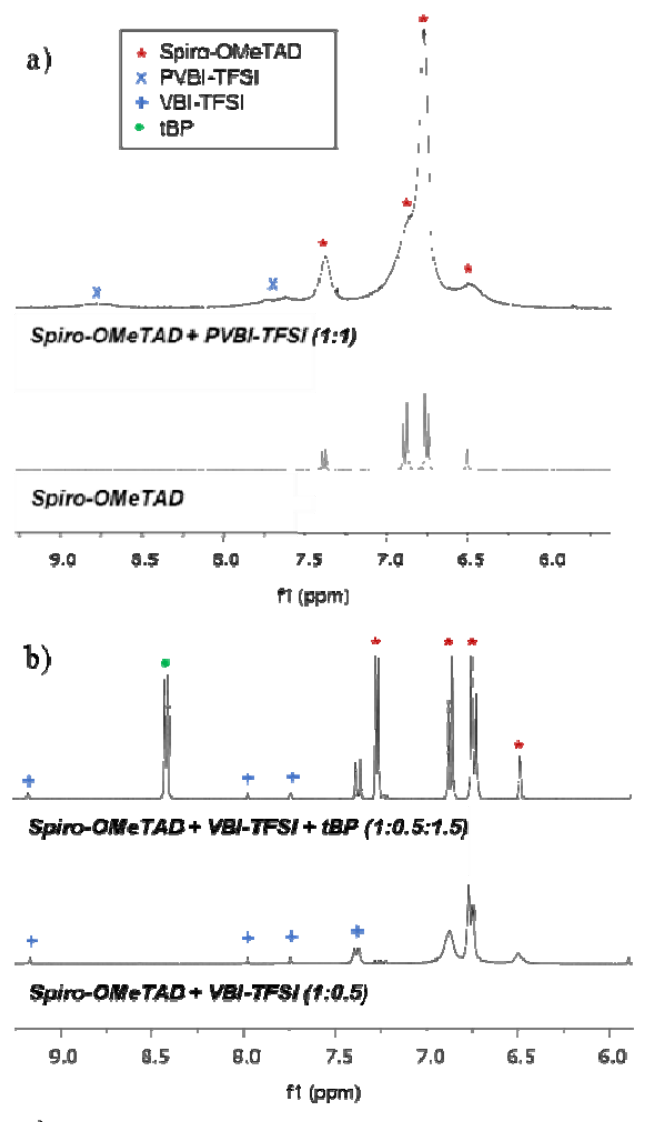

c)

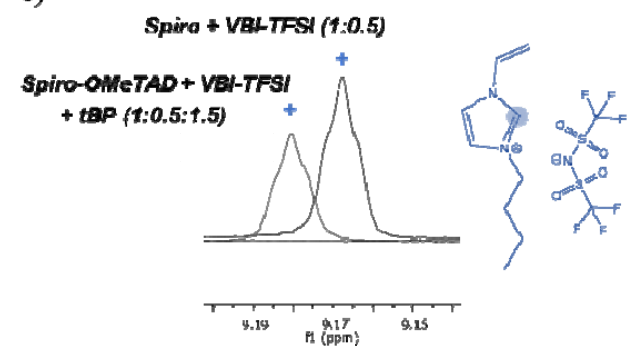

Figure 5. ${ }^{1} \mathrm{H} 400 \mathrm{~Hz}$ NMR spectra of a) spiro-OMeTAD, before and after PVBI-TFSI incorporation, and b) modification of spiro-VBI-TFSI spectrum through addition of $\mathrm{tBP}$ in d8-THF. c) Focus on the signal of the most acidic imidazolium proton of $\mathrm{VBI}^{+}$. 


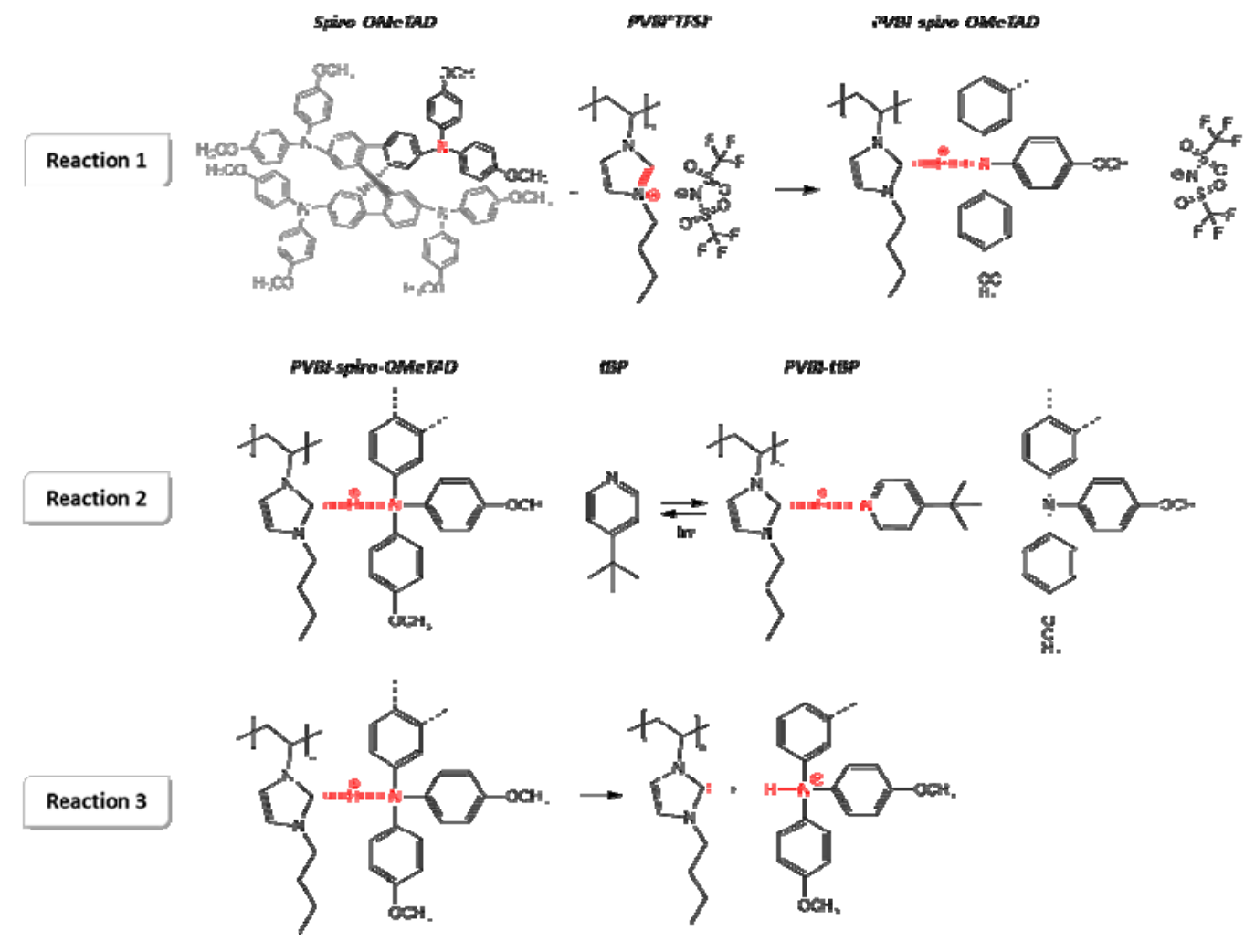

Figure 6. Proposed doping mechanism of spiro-OMeTAD by PVBI-TFSI in presence of $t$ BP.

Even if an interaction between the most acidic proton of the ionic liquid and the tertiary amine functions of the spiro-OMeTAD was justified by proton NMR, by a shift of the concerned proton and a quench of the aromatic protons of the spiro-OMeTAD, no evidence of the formation of carbenes was given.

Based on these observations and on previous works, ${ }^{28,29}$ we suppose that the most acidic proton on $\mathrm{PVBI}^{+}$is shared or even transferred to the basic nitrogen of the spiro-OMeTAD. This interaction was investigated by ${ }^{1} \mathrm{H}$ NMR spectroscopy. Usually, oxidation is evidenced by a broadening and/or a shifting of the associated protons and/or a decrease in signal intensity. Mixing spiroOMeTAD and PVBI-TFSI (1:1) in d8-THF led to a signal broadening of the aromatic protons of the spiro-OMeTAD without shifting, at $7.37,6.85,6.77$ and $6.48 \mathrm{ppm}$, and a slight decrease in intensity ( $v s$. the methoxy protons peak at $3.72 \mathrm{ppm}$ ) (Figure 5a). This peak broadening can stem from the formation of specific interactions between spiroOMeTAD and PVBI-TFSI, increasing the relaxation time of spiro-OMeTAD in solution, and/or to the formation of the spiro-OMeTAD ${ }^{+}$radical cation. However, as the presence of the polymer can increase the viscosity of the medium and, as such, increase the relaxation time of spiroOMeTAD, the effects on spiro-OMeTAD were studied in detail using the monomer VBI-TFSI as a model reaction.

Solutions of spiro-OMeTAD containing 50, 25 and 13 mol.\% of VBI-TFSI were compared. The increase in VBITFSI concentration resulted in a clear broadening of the aromatic peaks $(7.3-6.5 \mathrm{ppm})$ of the spiro-OMeTAD, and in a downfield shift of the most acidic proton of VBI-TFSI (9.2 - 9.1 ppm) (Figure S8). The broadening and shifting of the spiro-OMeTAD peaks suggest, at least, the formation of a hydrogen bonding between the imidazolium and the amine center of the spiro-OMeTAD. The shift observed for the imidazolium is consistent with an increase in proton dissociation. Both observations evidence the reaction 1 of the mechanism (Figure 6).

Secondly, a similar study was carried out to evidence the interactions between VBI-TFSI and $t$ BP (Figure S9). When increasing VBI-TFSI concentration, only a shift of the VBI-TFSI peak in the $9.15 \mathrm{ppm}$ region was observed. The unmodified peaks of $t \mathrm{BP}$ suggest that $\mathrm{VBI}+$ had weaker interactions with $t \mathrm{BP}$ than with spiro-OMeTAD. A weak hydrogen bonding is still considered between $\mathrm{VBI}+$ and the amine of $t \mathrm{BP}$ regarding the shift of the VBI-TFSI peak. When adding $t \mathrm{BP}$ to the spiro-OMeTAD doped with VBITFSI, the large peaks of the spiro-OMeTAD got their initial sharp shape back (Figure 5b). In addition, the most acidic proton of $\mathrm{VBI}^{+}$shifted towards upper fields, suggesting that $\mathrm{VBI}^{+}$stopped interacting with spiroOMeTAD in favor of interacting with $t \mathrm{BP}$ (Figure $5 \mathrm{c}$ ). An explication is the bigger size of spiro-OMeTAD making VBI-H-spiro-OMeTAD less stable than VBI-H- $t$ BP. In this way, in presence spiro-OMeTAD and $t \mathrm{BP}, \mathrm{VBI}-\mathrm{TFSI}$ preferentially weakly interacts with $t \mathrm{BP}$. Meanwhile, according to absorption spectroscopy experiments (Figure $4 \mathrm{~b}$ ), under light exposure, interactions with the most photoreactive material, i.e. spiro-OMeTAD is favored at the 
expense of $t \mathrm{BP}$. This is illustrated by the reaction 2 (Figure 6).

With further intense and longer light exposure, the $\mathrm{PVBI}^{+}$ proton could be transferred to spiro-OMeTAD to form PVBI carbene and spiro-OMeTAD-NH ${ }^{+}$. To detect the presence of carbenes, ${ }^{13} \mathrm{C}$ NMR was performed on the 25 mol.\% solution (Figure S10). 1.5 molar equivalent of $\mathrm{CS}_{2}$ were added to trap the carbenes. Carbenes should be evidenced by a shift of the "h" peak of VBI-TFSI at 136 ppm towards around $250 \mathrm{ppm}$. However, this signal remains at the same position upon the addition of spiroOMeTAD. Thus $\mathrm{VBI}^{+}$cannot protonate spiro-OMeTAD in solution. Nonetheless, it is noteworthy that the reaction 3 of the mechanism is not excluded to happen in thin film.

\section{CONCLUSION}

LiTFSI dopant was successfully substituted by PVBI-TFSI polyelectrolyte in spiro-OMeTAD. PVBI-TFSI was able to considerably increase spiro-OMeTAD conductivity by four orders of magnitude, up to $1.9 \times 10^{-3}{\mathrm{~S} . \mathrm{cm}^{-1}}^{\text {. Perovskite }}$ solar cells with PVBI-TFSI-doped spiro-OMeTAD showed power conversion efficiency as high as $20.3 \%$, as compared to $18.4 \%$ for devices incorporating LiTFSI-doped spiro-OMeTAD, with remarkably reduced hysteresis and high reproducibility. These improved PV performances were attributed to the higher conductivity of the spiroOMeTAD and to the defect-less morphology of the HTM layer. Up to now, this is the best PSC performance reported so far with an organic dopant in spiro-OMeTAD. The proposed doping mechanism involves a proton transfer from $\mathrm{PVBI}^{+}$to spiro-OMeTAD. This study demonstrates the great potential of $\mathrm{N}$-heterocyclic poly(ionic liquid)s to further improve PV performances of lithium-free PSCs via efficient HTM p-doping. The device stability of PSCs involving the polymeric dopant is under investigations.

\section{ASSOCIATED CONTENT}

Supporting Information. NMR and SEC characterizations of PVBI-TFSI, complementary UV-visible, NMR, AFM, PL, I$\mathrm{V}$ conductivity and photovoltaic data. This material is available free of charge via the Internet at http://pubs.acs.org.

\section{AUTHOR INFORMATION}

\section{Corresponding Author}

* E-mail: georges.hadziioannou@enscbp.fr (G.H.)

* E-mail: csegawa@mail.ecc.u-tokyo.ac.jp (H.S.)

* E-mail: thierry.toupance@u-bordeaux.fr (T.T.)

\section{Author Contributions}

All authors have given approval to the final version of the manuscript.

\section{ACKNOWLEDGMENT}

This work was performed within the framework of LIA NextPV. IDEX Bordeaux is thanked for PhD funding to CG. We are also thankful to ELORPrintTec ANR-10-EQPX-28-o1 and to LCPO/Arkema/ANR INDUSTRIAL CHAIR
"HOMERIC" ANR-13-CHIN-ooo2-01. The authors thank the New Energy and Industrial Technology Development Organization (NEDO) and Ministry of Economy, Trade and Industry in Japan for their financial supports.

\section{REFERENCES}

(1) Ponseca, C. S., Savenije, T. J., Abdellah, M., Zheng, K., Yartsev, A., Pascher, T., Harlang, T., Chabera, P., Pullerits, T., Stepanov, A., Wolf, J.-P. and Sundström, V. Organometal Halide Perovskite Solar Cell Materials Rationalized: Ultrafast Charge Generation, High and Microsecond-Long Balanced Mobilities, and Slow Recombination. J. Am. Chem. Soc. 136, 5189-5192, doi:10.1021/ja412583t (2014).

(2) Stranks, S. D., Eperon, G. E., Grancini, G., Menelaou, C., Alcocer, M. J. P., Leijtens, T., Herz, L. M., Petrozza, A. and Snatih, H. J. Electron-Hole Diffusion Lengths Exceeding $1 \mathrm{Mi}-$ crometer in an Organometal Trihalide Perovskite Absorber. Science 342, 341 doi:10.1126/science.1243982 (2013).

(3) Gao, P., Gratzel, M. and Nazeeruddin, M. K. OrganoHalide Lead Perovskites for Photovoltaic Applications. Energy Environ. Sci. 7, 2448-2463, doi:10.1039/C4EEoo942H (2014).

(4) Kojima, A., Teshima, K., Shirai, Y. and Miyasaka, T. Organometal Halide Perovskites as Visible-Light Sensitizers for Photovoltaic Cells. J. Am. Chem. Soc. 131, 6050-6051, doi:10.1021/ja809598r (2009).

(5) Yang, W. S., Park, B.-W., Jung, E. H., Jeon, N. J., Kim, Y. C., Lee, D. U., Shin, S. S., Seo, J., Kim, E. K., Noh, J. H. and Seok, S. I. Iodide Management in Formamidinium-Lead-Halidebased Perovskite Layers for Efficient Solar Cells. Science 356, 1376-1379 doi:10.1126/science.aan2301 (2017).

(6) NREL. Best Research-Cell Efficiencies, $<$ https://www.nrel.gov/pv/assets/pdfs/pv-efficiencies-02-o82019.pdf>

(7) Zhao, Y., Tan, H., Yuan, H., Yang, Z., Fan, J. Z., Kim, J., Voznyy, O., Gong, X., Quan, L. N., Tan, C. S., Hofkens, J., Yu, D., Zhao, Q. and Sargent E. H. Perovskite Seeding Growth of Formamidinium-Lead-Iodide-based Perovskites for Efficient and Stable Solar Cells. Nat. Commun. 9, 1607, doi:10.1038/s41467-01804029-7 (2018).

(8) Shin, S. S., Yeom, E. J., Hur, S., Kim, M. G. Im, J., Seo, J., Noh, J. H. and Seok, S. I. Colloidally Prepared La-doped BaS$\mathrm{nO}_{3}$ Electrodes for Efficient Photostable Perovskite Solar Cells. Science 356, 167-171 doi:10.1126/science.aam6620 (2017).

(9) Saliba, M., Matsui, T., Domanski, K., Seo, J.-Y., Ummadisingu, A., Zakeeruddin, S. M., Correa-Baena, J.-P., Tress, W. R., Abate, A., Hagfeldt, A. and Grätzel, M.. Incorporation of Rubidium Cations into Perovskite Solar Cells Improves Photo$\begin{array}{llll}\text { voltaic } & \text { Performance. } & \text { Science } & \text { 354, }\end{array}$ doi:10.1126/science.aah5557 (2016).

(10) Wang, S., Yuan, W. and Meng, Y. S. SpectrumDependent Spiro-OMeTAD Oxidization Mechanism in Perovskite Solar Cells. ACS Appl. Mater. Interfaces 7, 24791-24798, doi:10.1021/acsami.5bo7703 (2015).

(11) Abate, A., Leitjens, T., Pathak, S., Teuscher, J., Avolio, R., Errico, M. E., Kirkpatrik, J., Ball, J. M., Docampo, P., McPherson, I. and Snaith, H. J. Lithium Salts as "Redox Active" p-Type Dopants for Organic Semiconductors and their Impact in SolidState Dye-Sensitized Solar Cells. Phys. Chem. Chem. Phys., 15, 2572, doi:10.1039/c2cp44397j (2013).

(12) Berhe, T. A., Su, W.-N., Chen, C.-H., Pan, C.-J., Cheng, J.-H., Chen, H.-M., Tsai, M.-C., Chen, L-Y., Dubale, A. A. and Hwang, B.-J. Organometal Halide Perovskite Solar Cells: Degradation and Stability. Energy Environ. Sci. 9, 323-356, doi:10.1039/C5EEo2733K (2016).

(13) Schloemer, T. H., Christians, J. A., Luther, J. M. and Sellinger, A., Doping Strategies for Small Molecule Organic 
Hole-Transport Materials: Impacts on Perovskite Solar Cell Performances and Stability. Chem. Sci. 10, 1904-1935 doi:10.1039/C8SC05284K (2019).

(14) Koh, T. M., Fu, K., Fang, Y., Chen, S., Sum, T. C., Mathews, N., Mhaisalkar, S. G., Boix, P. P. and Baikie, T. Formamidinium-Containing Metal-Halide: An Alternative Material for Near-IR Absorption Perovskite Solar Cells. J. Phys. Chem. C 118, 16458-16462, doi:10.1021/jp411112k (2014).

(15) Noh, J. H., Jeon, N. J., Choi, Y. C., Nazeeruddin, M. K., Grätzel, M. and Seok S. I. Nanostructured $\mathrm{TiO}_{2} / \mathrm{CH}_{3} \mathrm{NH}_{3} \mathrm{PbI}_{3}$ Heterojunction Solar Cells Employing Spiro-OMeTAD/CoComplex as Hole-Transporting Material. J. Mater. Chem. A 1, 11842-11847, doi:10.1039/C3TA12681A (2013).

(16) Krishnamoorthy, T., Kunwu, F., Boix, P. P., Li, H., Koh, T. M., Leong, W. L., Powar, S., Grimsdale, A., Grätzel, M., Mathews, N. and Mhaisalkar, S. G. A Swivel-Cruciform Thiophene based Hole-Transporting Material for Efficient Perovskite Solar Cells. J. Mater. Chem. A 2, 6305-6309, doi:10.1039/C4TA00486H (2014).

(17) Pellaroque, A., Noel, N. K., Habisreutinger, S. N., Zhang, Y., Barlow, S., Marder, S. R. and Snaith, H. J. Efficient and Stable Perovskite Solar Cells Using Molybdenum Tris(dithiolene)s as p-Dopants for Spiro-OMeTAD. ACS Energy Lett. 2, 2044-2050, doi:10.1021/acsenergylett.7boo614 (2017).

(18) Li, M., Wang, Z.-K., Yang, Y.-G., Hu, Y., Feng, S.-L., Wang, J.-M., Gao, X.-Y. and Liao, L.-S. Copper Salts Doped Spiro-OMeTAD for High-Performance Perovskite Solar Cells. Adv. Energy Mater. 6, 1601156, doi:10.1002/aenm.201601156 (2016).

(19) Nguyen, W. H., Bailie, C. D., Unger, E. L. and McGehee, M. D. Enhancing the Hole-Conductivity of Spiro-OMeTAD without Oxygen or Lithium Salts by Using Spiro(TFSI)2 in Perovskite and Dye-Sensitized Solar Cells. J. Am. Chem. Soc. 136, 10996-11001, doi:10.1021/ja504539w (2014).

(20) Bach, U. Solid-State Dye-Sensitized Mesoporous $\mathrm{TiO}_{2}$ Solar Cells, Ph.D. Thesis. Ecole Polytechnique Fédérale de Lausanne, (2000).

(21) Xu, M., Rong, Y., Ku, Z., Mei, A., Li, X. and Han, H. Improvement in Solid-State Dye Sensitized Solar Cells by p-Type Doping with Lewis Acid $\mathrm{SnCl}_{4}$. J. Phys. Chem. C 117, 2249222496, doi:10.1021/jp406506d (2013).

(22) Chen, D. Y., Tseng, W.-H., Liang, S.-P., Wu, C.-I., Hsu, C.-W., Chi, Y., Hung, W.-Y. and Chou, P.-T. Application of $\mathrm{F}_{4}$ TCNQ Doped Spiro-MeOTAD in High Performance Solid State Dye Sensitized Solar Cells. Phys. Chem. Chem. Phys. 14, 1168911694, doi:10.1039/c2cp41855j (2012).

(23) Ye, T., Wang, J., Chen, W., Yang, Y. and He, D. Improved Performance and Reproducibility of Perovskite Solar Cells by Well-Soluble Tris(pentafluorophenyl)borane as a p-Type Dopant. ACS Appl. Mater. Interfaces 9, 17923-17931, doi:10.1021/acsami.7bo2969 (2017).

(24) Qiuju Liu, L. F., Zhang, Q., Zhou, A., Wang, B., Bai, H., Tian, Q., Fan, B. and Zhang, T. Benzoyl Peroxide as an Efficient Dopant for Spiro-OMeTAD in Perovskite Solar Cells. ChemSusChem, 10, 3098-3104 doi:10.1002/cssc.201700872 (2017).

(25) Luo, J., Xia, J., Yang, H., Chen, L., Wan, Z., Han, F., Malik, H. A., Zhu, X. and Jia, C. Toward High-Efficiency, HysteresisLess, Stable Perovskite Solar Cells: Unusual Doping of a HoleTransporting Material using a Fluorine-Containing Hydrophobic Lewis Acid. Energy Environ. Sci. 11, 2035-2045 doi:10.1039/c8eeooo36k (2018).

(26) Seo, J.-Y., Kim, H.-S., Akin, S., Stojanovic, M., Simon, E., Fleischer, M., Hagfeldt, A., Zakeeruddin, S. M. and Grätzel, M. Novel p-Dopant Toward Highly Efficient and Stable Perovskite Solar Cells. Energy Environ. Sci. 11, 2985-2992, doi:10.1039/C8EEo150oG (2018).

(27) Abate, A., Hollman D. J., Teuscher, J., Pathak, S., Avolio, R., D'Errico, G., Vitiello, G., Fantacci, S. and Snaith, H. J.
Protic Ionic Liquids as p-Dopant for Organic Hole Transporting Materials and Their Application in High Efficiency Hybrid Solar Cells. J. Am. Chem. Soc. 135, 13538-13548, doi:10.1021/ja40623of (2013).

(28) Zhang, H., Shi, Y., Yan, F., Wang, L., Wang, K., Xing, Y., Dong, Q. and Ma, T. A Dual Functional Additive for the HTM Layer in Perovskite Solar Cells. Chem. Commun. 50, 5020-5022, doi:10.1039/C 3 CC49458F (2014).

(29) Caliò, L., Salado, M., Kazim, S. and Ahmad, S. A. Generic Route of Hydrophobic Doping in Hole Transporting Material to Increase Longevity of Perovskite Solar Cells. Joule, doi:https://doi.org/10.1016/j.joule.2018.06.012 (2018).

(3о) Li, Z., Xiao, C., Yang, Y., Harvey, S. P., Kim, D. H., Christians, J. A., Yang, M., Schulz, P., Nanayakkara, S. U., Jiang, C.-S., Luther, J. M., Berry, J. J., Beard, M. C., Al-Jassim, M. M. and Zhu, K. Extrinsic Ion Migration in Perovskite Solar Cells. Energy Environ. Sci. 10, 1234-1242, doi:10.1039/C 7 EEoo358G (2017).

(31) Tang, Z., Bessho, T., Awai, F., Kinoshita, T., Maitani, M. M., Jono, R., Murakami, T. N., Wang, H., Kubo, T., Uchida, S. and Segawa, H. Hysteresis-Free Perovskite Solar Cells Made of Potassium-Doped Organometal Halide Perovskite. Sci. Rep. 7, 12183, doi:10.1038/s41598-017-12436-x (2017).

(32) Tang, Z., Uchida, S., Bessho, T., Kinoshita, T., Wang, H., Awai, F., Jono, R., Maitani, M. M., Nakazaki, J., Kubo, T. and Segawa, H. Modulations of Various Alkali Metal Cations on Organometal Halide Perovskites and their Influence on Photovoltaic Performance. Nano Energy 45, 184-192, doi.org/10.1016/j.nanoen.2017.12.047 (2018).

(33) Magomedov, A., Kasparavicius, E., Rakstys, K., Paek, S Gasilova, N., Genevicius, K., Juska, G., Malinauskas, T., Nazeeruddin, M. K. and Getautis, V. Pyridination of Hole Transporting Material in Perovskite Solar Cells Questions the Long-Term Stability. J. Phys. Chem. C 6, 8874-8878 doi:10.1039/C8TC02242A (2018).

(34) Ishii, H., Sugiyama, K., Ito, E. and Seki, K. Energy Level Alignment and Interfacial Electronic Structures at Organic/Metal and Organic/Organic Interfaces. Adv. Mater. 11, 605625, doi:10.1002/(sici)1521-4095(199906)11:8<605::aidadma6o5>3.o.co;2-q (1999).

(35) Almosni, S., Cojocaru, L., Li, D., Uchida, S., Kubo, T. and Segawa, H. Tunneling-Assisted Trapping as one of the Possible Mechanisms for the Origin of Hysteresis in Perovskite Solar Cells. Energy Technol. 5, 1767-1774, doi:10.1002/ente.201700246 (2017).

(36) Abdi-Jalebi, M., Andaji-Garmaroudi, Z., Cacovich, S., Stavrakas, C., Philippe, B., Richter, J. M., Alsari, M., Booker, E. P., Hutter, E. M., Pearson, A. J., Liliu, S., Savenije, T. J., Rensmo, H., Divitini, G., Ducati, C., Friend, R. H. and Stranks, S. D. Maximizing and Stabilizing Luminescence from Halide Perovskites with Potassium Passivation. Nature 555, 497, doi:10.1038/nature25989 (2018).

(37) de Frémont, P., Marion, N. and Nolan, S. P. Carbenes: Synthesis, Properties, and Organometallic Chemistry. Coord. Chem. Rev. 253, 862-892, doi:https://doi.org/10.1016/j.ccr.2008.05.018 (2009). 
Table of Contents

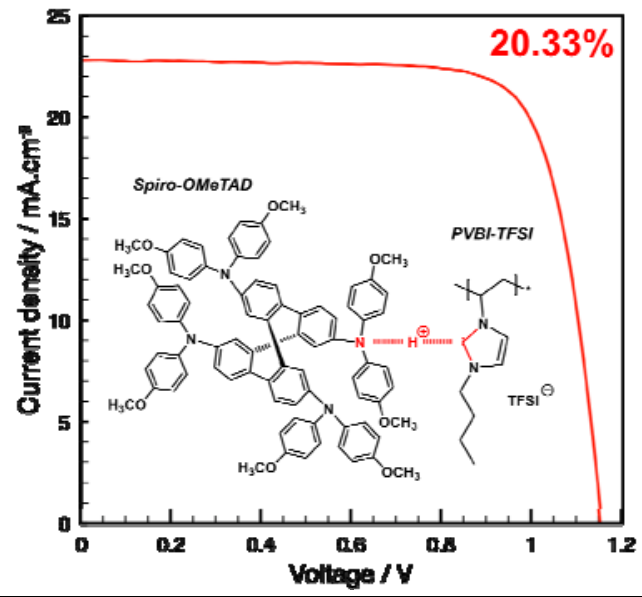

